prognosis signature group compared with the good-prognosis signature group was 5.1, and the hazard ratio for overall survival was 8.6. van de Vijer et al. compare their classification with that made by standard consensus criteria, and suggest that the current criteria misclassify many patients, which results in some patients getting unnecessary treatment and others missing out on adjuvant treatment that would be beneficial. This has important implications for the future of breast cancer management, especially if the prediction technique is validated in older patients who have more advanced disease.

(i) References and links ORIGINAL RESEARCH PAPER Van de Vijver, M. J. et al. A gene-expression signature as a predictor of survival in breast cancer. N. Engl. J. Med. $\mathbf{3 4 7}$, 1999-2009 (2002)

FURTHER READING van't Veer, L. J. et al. Gene expression profiling predicts clinical outcome of breast cancer. Nature 415, 530-536 (2002) WEB SITE

Marc van de Vijver's lab:

http://www.nki.nl/nkidep/vdvijver/index.htm

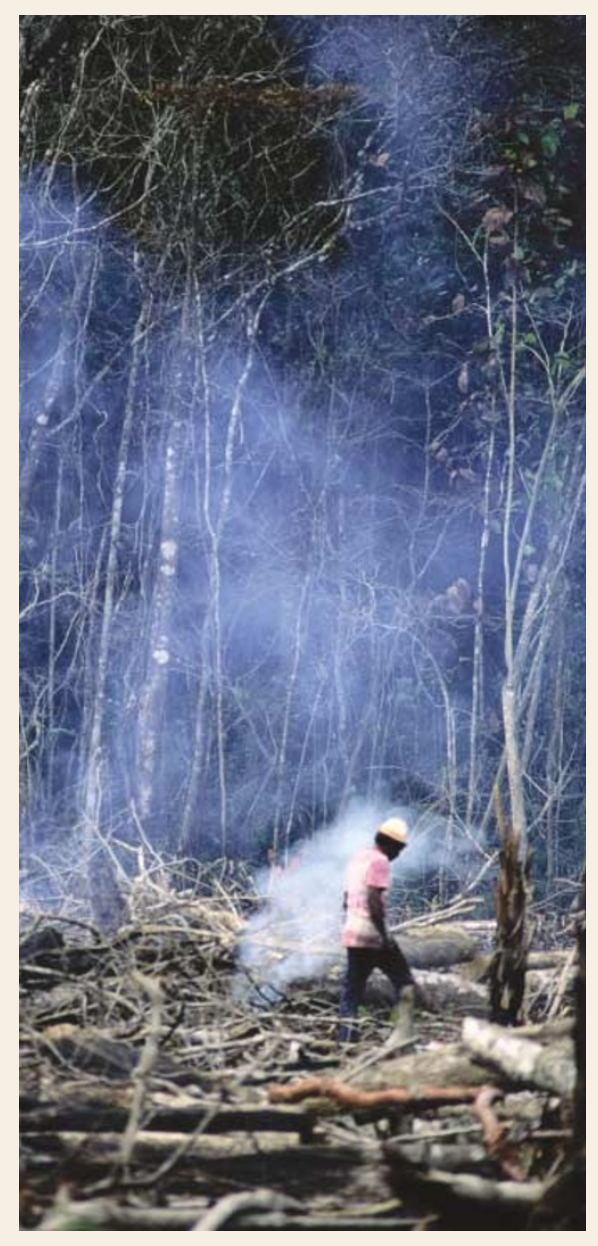

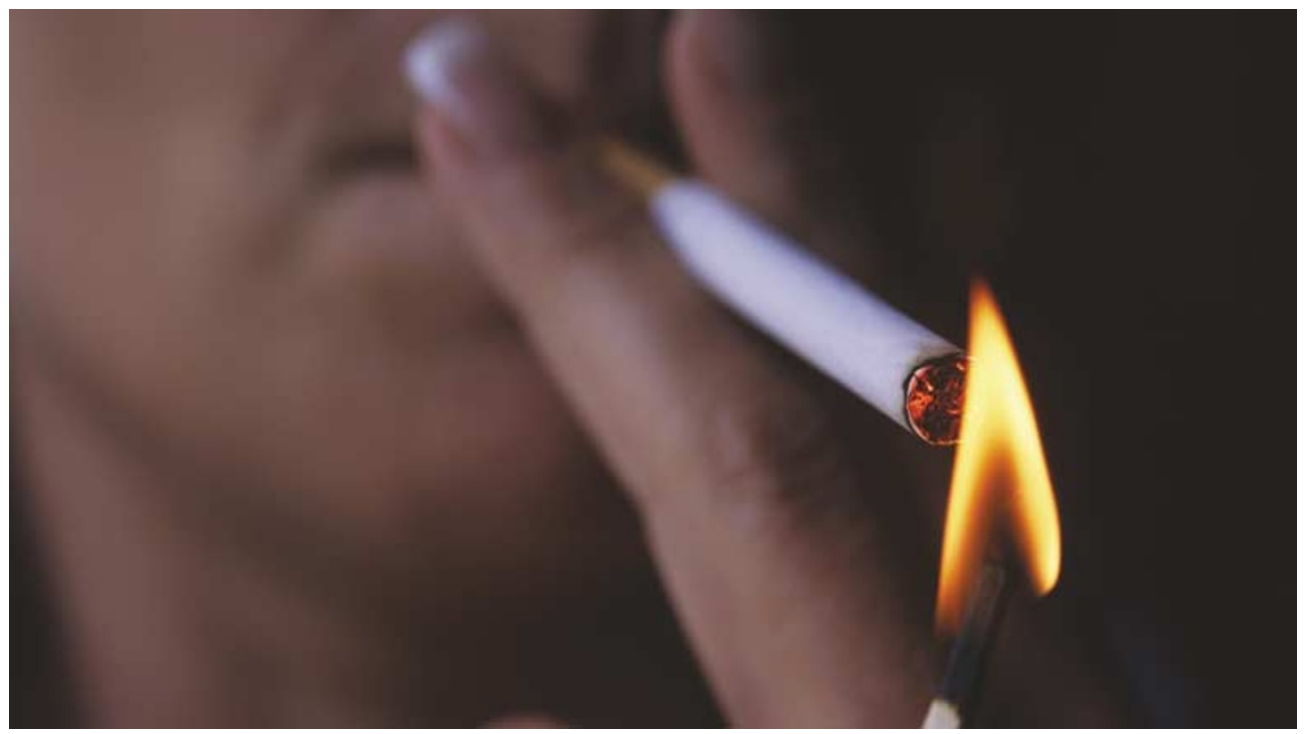

CARCINOGENESIS

\section{Double whammy}

Tobacco smoke contains more than 40 carcinogenic chemicals, most of which are believed to cause lung cancer through the induction of DNA damage. In the January issue of The Journal of Clinical Investigation, Kip West et al. show that tobacco also has a direct biochemical effect on cells through activation of the anti-apoptotic signalling protein AKT.

West et al. began investigating how lung epithelial cells that have undergone tobacco-induced DNA damage evade apoptosis and eventually become resistant to chemotherapeutic drugs. The anti-apoptotic protein AKT was previously observed to be constitutively activated in non-small-cell lung cancer cell lines. This serine/threonine kinase controls several cellular processes, including glucose metabolism, cell-cycle progression and apoptosis.

To test whether tobacco-derived carcinogens could activate this pathway in human lung epithelial cells, West et al. examined normal human bronchial epithelial cells derived from large airways, which can become squamous-cell carcinomas, and small airway epithelial cells, which can become adenocarcinomas. These cells were treated with two components of tobacco - nicotine, which is the addictive compound of tobacco and a precursor to many carcinogens, and 4-(methylnitrosamino)-1(3-pyridyl)-1-butanone (NNK), which is a potent tobacco-specific carcinogen.

Nicotine and NNK both induced AKT phosphorylation, which is required for activation, in these cell lines. This led to phosphorylation of downstream targets that control the cell cycle and protein translation, such as GSK3, forkhead transcription factor family (FKHR), eukaryotic translation initiation factor $4 \mathrm{E}$ (EIF4B) and the ribosomal kinase protein S6. After treatment, cell adhesion and dependence on exogenous growth factors two indicators of transformation - GSK3 were reduced . Most importantly, nicotine/NNK activation of this pathway increased cell survival after treatment with etoposide, ultraviolet irradiation or $\mathrm{H}_{2} \mathrm{O}_{2}$. So, this might be a mechanism by which lung cancer cells become resistant to chemotherapy.

Nicotine and NNK had similar effects in vivo, as treatment of mice with these agents led to AKT phosphorylation in airway epithelial cells and the development of aggressive lung tumours. AKT phosphorylation was also detected in ten lung cancer specimens derived from smokers.

But how do nicotine and NNK activate AKT signalling? They both bind to specific subgroups of nicotinic acetylcholine receptors (AchRs) that are expressed by bronchial epithelial and endothelial cells. West et al. showed that treatment of bronchial epithelial cells with inhibitors of these receptors blocked nicotine- and NNK-induced AKT phosphorylation, whereas an AchR agonist increased AKT phosphorylation. So, nicotine and NNK seem to activate AKT by signalling through an AchR, although other undiscovered signalling pathways might exist.

Kinetic studies in the cells showed that AKT activation preceded DNA-damage induction, so nicotine and NNK might deliver a 1-2 punch, exerting not only genotoxic effects but also protecting cells from subsequent induction of apoptosis. Nicotine has also been shown to stimulate endothelial-cell proliferation and angiogenesis, so the authors suggest that nicotine replacement therapy for smoking cessation could have long-term carcinogenic effects. GlaxoSmithKline and Pharmacia, which make smoking cessation products, have both issued press releases stating that there is no clinical evidence that nicotine replacement therapy causes cancer.

Kristine Novak

\section{(Q) References and links}

ORIGINAL RESEARCH PAPER West, K. A. et al. Rapid Akt activation by nicotine and a tobacco carcinogen modulates the phenotype of normal human airway epithelial cells. J. Clin. Invest. 111, 81-90 (2003) FURTHER READING Schuller, H. M. Mechanisms of smoking-related lung and pancreatic adenocarcinoma development. Nature Rev. Cancer 2, 455-463 (2002)

WEB SITE

Phillip Dennis's lab: http://ccr.cancer.gov/Staff/Staff.asp?StafflD=572 